\title{
Effectiveness Of Continuous Versus Pulsed Short-Wave Diathermy in the Management Of Knee
}

Osteoarthritis: A Pilot Study

$\underline{\text { Selin Ozen }}{ }^{1}$, Ekin Başak Doganci ${ }^{1}$, Ayla Ozyuvali ${ }^{1}$, Alper Doganci ${ }^{1}$, Ayşe Peyman Yalcin ${ }^{1}$ ${ }^{1}$ Department of Physical and Rehabilitation Medicine, Ankara University Faculty of Medicine, Ankara, Turkey

Introduction: Knee osteoarthritis (KOA) is one of the most common forms of arthritis in the Western world, with a prevalence of 10 to $15 \%$ in adults over 60 years of age. KOA results in chronic joint pain, muscle weakness and loss of function. Diathermy is a form of physical therapy in which deep heating of tissues is accomplished by the use of high-frequency electrical current. In 1891 Nikola Tesla first noted that heat resulted from irradiation of tissue with highfrequency alternating current and pointed out its possible medical uses. In the 1930s Short-wave diathermy (SWD), its physical properties and its beneficial therapeutic uses became a popular topic of discussion. Despite SWD being one of the oldest forms of electrotherapeutic modalities used by physical therapists in the conservative treatment of knee osteoarthritis (KOA), the Osteoarthritis Research Society International guideline for the non-surgical management of KOA did not feature SWD. The reason for this maybe that even though SWD treatment appears to be effective for decreasing pain and increasing muscle strength in patients with $\mathrm{KOA}$, there is no consensus on outcomes with continuous (thermic) versus pulsed (athermic) SWD.

Purpose: To compare the effects of continuous (thermic) versus pulsed (athermic) SWD on pain, function and activity in women with KOA

Method: 27 female patients presenting to our outpatient clinic, between the ages of 50-65 with complaints of bilateral knee pain and a diagnosis of KOA according to the American College of Rheumatology clinical criteria for the classification of osteoarthritis of the knee, were randomised into two groups. Only patients with radiographic evidence of grade 2-3 osteoarthritis in the tibiofemoral compartment on bilateral anteroposterior and lateral knee radiographs according to the Kellgren - Lawrance scale were included in the study. Those who had received physical therapy to the knee joint over the past 6 months, those with reduction of range of motion of the knee, those with known hip/knee/ankle pathologies, those with an inflammatory arthropathy, those who had a history of knee trauma or knee intervention over the past six months, those with metal implants, a cardiac pacemaker or malignancy were excluded from the study.

Sociodemographic data included age, weight, body mass index (BMI), symptom duration, job status, level of education. Drug treatment including use of analgesics was questioned. Those on non steroidal anti-inflammatory drugs (NSAIDS) were switched to diclofenac 75mg SR od one week prior to treatment.

Patients were randomized into one of two treatment groups by the same physician. Power analysis of the study was carried out based on an improvement of 2 units on the VAS scale at the evaluation one month post treatment being considered as a significant improvement. The Power of the study based on this and having 45 patients in each group (a total of 90 patients) is $80 \%$ with a $5 \%$ type one error. It was predicted that the VAS scores one month post treatment would show 3 standard deviations of change and that the drop out rate $15 \%$. The patients were randomised into two groups using block randomisation.

SWD electromagnetic radiation at a frequency of $27.12 \mathrm{MHz}$ was applied in a continuous mode (CSWD) in group one and pulsed mode (PSWD) in group two. Treatment was administered using the Curapuls 419 SWD machines (Enraf-Nonius, Delft, The Netherlands). No other physical therapy was given. Sessions lasted fifteen minutes, on five consecutive days per week for a total of three weeks. Written informed consent was obtained from all patients prior to commencing evaluation and treatment. Patients were assessed before treatment, after treatment and at one month post treatment. All assessments were carried out by the same physician who was blind to the treatment received by the patient. Outcome measures included the visual analogue scale (VAS), Western Ontario and Mcmaster University Osteoarthritis Index (WOMAC) and a six minute walking test (6MWT).

The data was analysed using SPSS for Windows (IBM ${ }^{\circledR}$ SPSS $^{\circledR}$ statistics version 22). Chi squared and Fischer's exact tests were used for comparison data. When comparing the age of patients, BMI, symptom duration, VAS, WOMAC between the two groups, the student's T test was used for comparison of parametric data and Mann Whitney U test for non parametric data. When comparing parametric VAS, WOMAC and 6MWT data of group one and group two prior to, post and one month post treatment, repeated measures analysis of variance (ANOVA) was used; non parametric data was compared using the Friedman Test. When a significant difference was found between two values, this was further evaluated using a post hoc multiple comparison (Bonferroni test). p $<0.05$ was considered statistically significant.

\section{Results:}

The mean age of the patients treated with continuous SWD (group 1) was 57.9 \pm 5.0 (med. 52, range $52-65$ ), those treated with pulsed SWD (group 2) was $54.8 \pm 4.4$ (med. 56.5 , range $49-60$ ). BMI was $33.6 \pm 4.1$ in group one and $34.7 \pm 4.8$ in group two. There was no statistical difference between groups in terms of age and BMI ( $>0.05)$. Those given continuous SWD had symptoms of KOA on average 87.6 months (range 4-180) and those given pulsed SWD had symptoms of KOA on average 36.6 months (range 1-120) $\mathrm{p}=0.021$. There was no significant difference in VAS $(\mathrm{p}=0.839)$ and 6MWT $(\mathrm{p}=0.367)$ scores prior to treatment.

Statistically significant difference was found in the pulsed SWD group regarding the VAS score over time $(\mathrm{p}=0.003<0.01)$. This was not the case for the continuous SWD group ( $>>0.05)$. (Table 1$)$

Table 1: VAS scores before and after treatment

\begin{tabular}{|c|c|c|c|c|}
\hline & $\begin{array}{l}\text { Pre treatment } \\
6 \mathrm{MWT}\end{array}$ & $\begin{array}{l}\text { Post treatment } \\
\text { 6MWT }\end{array}$ & $\begin{array}{c}1 \text { month post } \\
\text { treatment } \\
\text { 6MWT }\end{array}$ & \\
\hline $\begin{array}{l}\text { Continuous } \\
\text { SWD (n=11) } \\
\text { Mean } \pm \text { SD } \\
\text { Range }\end{array}$ & $\begin{array}{c}352.27 \pm 67.36 \\
(250-440)\end{array}$ & $\begin{array}{c}361.00 \pm 70.86 \\
(0-100)\end{array}$ & $\begin{array}{c}365.91 \pm 73.88 \\
(260-483)\end{array}$ & $\begin{array}{l}F=1.197 \\
p=0.300\end{array}$ \\
\hline $\begin{array}{l}\text { Pulsed SWD } \\
(\mathbf{n}=16) \\
\text { Mean } \pm \text { SD } \\
\text { Range }\end{array}$ & $\begin{array}{c}323.25 \pm 88.31 \\
(80-460)\end{array}$ & $\begin{array}{c}344.38 \pm 68.76 \\
(200-440)\end{array}$ & $\begin{array}{c}363.56 \pm 87.78 \\
(200-504)\end{array}$ & $\begin{array}{l}F=8.594 \\
p=0.010\end{array}$ \\
\hline
\end{tabular}

\begin{tabular}{|l|c|c|c|c|}
\hline & $\begin{array}{c}\text { Pre treatment } \\
\text { VAS }\end{array}$ & $\begin{array}{c}\text { Post treatment } \\
\text { VAS }\end{array}$ & $\begin{array}{c}\text { 1 month post } \\
\text { treatment VAS }\end{array}$ & \\
\hline $\begin{array}{l}\text { Continuous } \\
\text { SWD (n=11) }\end{array}$ & & & & \\
$\begin{array}{l}\text { Mean } \pm \text { SD } \\
\text { Range }\end{array}$ & $\begin{array}{c}77.3 \pm 20.0 \\
(50-100)\end{array}$ & $\begin{array}{c}40.0 \pm 27.9 \\
(0-100)\end{array}$ & $\begin{array}{c}49.1 \pm 28.9 \\
(10-100)\end{array}$ & $\begin{array}{c}\mathrm{X}^{2}=5.707 \\
\mathrm{p}=0.058\end{array}$ \\
\hline $\begin{array}{l}\text { Pulsed SWD } \\
(\mathbf{n}=16)\end{array}$ & $75.0 \pm 21.68$ & $43.8 \pm 26.2$ & $49.1 \pm 28.9$ & $\mathrm{X}^{2}=11.660$ \\
$\begin{array}{l}\text { Mean } \pm \text { SD } \\
\text { Range }\end{array}$ & $(40-100)$ & $(0-90)$ & $(0-100)$ & $\mathrm{p}=0.003<0.01$ \\
\hline
\end{tabular}

There was a statistically significant improvement in the 6MWT in group 2 one month after treatment when compared to pre-treatment values $(\mathrm{p}=0.031)$. (table3). There was no statistically significant change in WOMAC scores, including the subgroups, in either group ( $p>0.05)$.

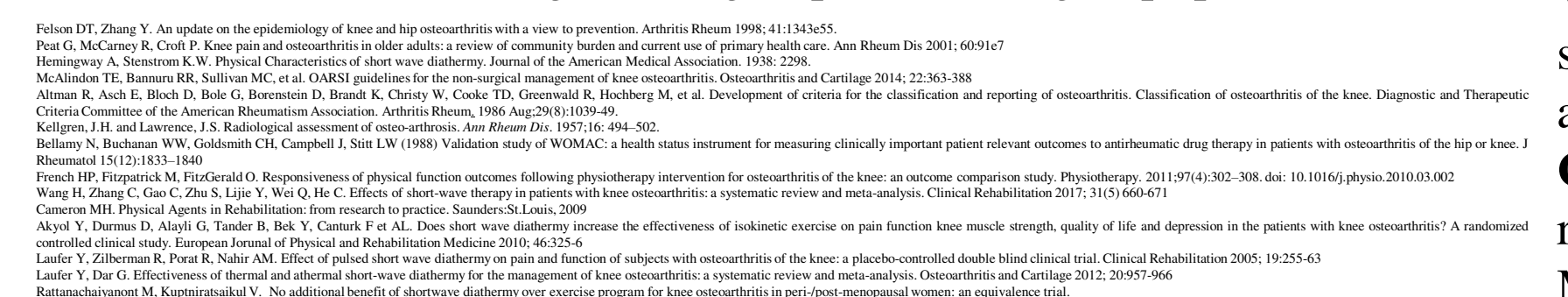

Discussion: The findings of this study suggest that pulsed SWD has a positive effect on both reducing pain and improving walking speed. The reduction in pain following treatment with pulsed SWD as opposed to continuous SWD observed in this study is in keeping with the pooled date of the systematic review by Wang et al. Furthermore, they found that short wave therapy was more effective in treating women with KOA. We believe the ability of pulsed SWD to reduce inflammation, synovial thickness results in a reduction in joint stiffness and pain and this maybe the reason why its ability to reduce pain was superior to continuous SWD. In contrast, some past studies have shown that the positive effect on pain perception is achieved only when the treatment involves at least some degree of thermal sensation but that despite this, the benefits of pain reduction are lost within 9-12 weeks of follow up post therapy.,

There was no significant improvement in physical function in either group as evaluated by the WOMAC. This, once again is in keeping with previous studies. ${ }^{11}$,

There was a significant improvement in walking distance in the pulsed SWD group one month post treatment. In contrast, Laufer at al. found no significant improvement in a three minute walking test prior to and following treatment with thermic and athermic SWD. ${ }^{12}$ Rattanachaiyanont et al. found trivial but statistical improvement in WOMAC and $100 \mathrm{~m}$ walking test in both exercise + continuous SWD and exercise + sham SWD groups suggesting that SWD does not prove to be superior to exercise alone.

Conclusion: Treatment of KOA with pulsed SWD appears to be more effective at reducing pain and improving walking distance when compared to continuous SWD. More patients must be recruited to the study in order to consolidate these findings. 